\title{
'Working out' mitochondrial calcium
}

The first report of mice lacking the mitochondrial calcium uniporter (MCU) - which transports intracellular calcium across the inner mitochondrial membrane - shows that calcium uptake by mitochondria during skeletal muscle work is a key determinant of the maximal power output. In contrast to the predictions of previous studies on the role of mitochondrial calcium, $\mathrm{Mcu}^{-/-}$mice were no different from wild-type mice in terms of basal metabolism or the susceptibility of tissues to cell death.

Finkel and colleagues used a gene trap strategy to prematurely truncate $\mathrm{Mcu}$, and confirmed lack of expression of MCU at the mRNA and protein levels. The $\mathrm{Mcu}^{-/-}$mice had normal overall body composition and no obvious abnormalities in mitochondrial number or morphology. By assessing purified mito-

chondria from skeletal muscle and

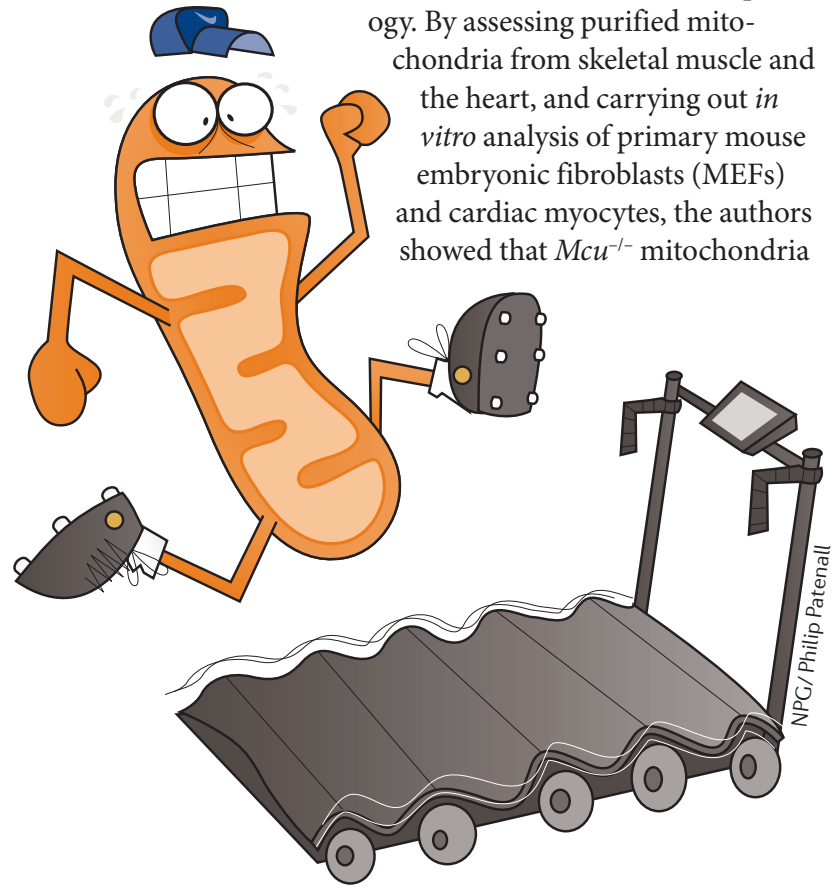

are unable to rapidly take up cytosolic calcium. Calcium uptake could be restored by reconstitution with wild-type, but not mutated, MCU.

Under basal conditions, there was no detectable difference between MEFs from $\mathrm{Mcu}^{-/-}$or wild-type mice in terms of oxygen consumption, as judged using various metabolic substrates, and they had similar maximal oxidative capacity. Furthermore, total body basal oxygen consumption was not altered in $\mathrm{Mcu}^{-/-}$mice. So, although previous studies have shown that mitochondrial calcium regulates the activity of metabolic enzymes, basal metabolism is not markedly altered in mice lacking MCU. The authors suggest that slower, low capacitance mechanisms compensate for mitochondrial calcium entry in $\mathrm{Mcu}^{-/-}$mice, which have resting mitochondrial calcium levels of $25 \%$ of wild-type mice.

MCU-dependent rapid entry of mitochondrial calcium might be a regulatory mechanism to increase ATP production in response to increased demand. In support of this, when skeletal muscle work load was increased by placing mice on a treadmill or assessing forearm grip strength, there was a significant decrease in the exercise capacity and strength of $\mathrm{Mcu}^{-/-}$mice. Mechanistically, decreased levels of calcium in the mitochondria of $\mathrm{Mcu}^{-1-}$ mice were associated with increased phosphorylation of the mitochondrial metabolic enzyme pyruvate dehydrogenase (PDH), the dephosphorylation of which is mediated by the calcium-sensitive phosphatase PDP1. The addition of calcium to wild-type, but not $\mathrm{Mcu}^{-/-}$, mitochondria led to a rapid reduction in $\mathrm{PDH}$ phosphorylation and hence increased metabolic activity.

The uptake of large amounts of mitochondrial calcium through MCU can induce cell death by causing the permeability transition pore (PTP) to open. Consistent with this, isolated $\mathrm{Mcu}^{-/-}$mitochondria showed no evidence of PTP opening after exposure to high levels of calcium. However, there was no difference between wild-type and $\mathrm{Mcu}^{-1-}$ MEFs in the kinetics or magnitude of cell death in response to a range of inducers in vitro. Similarly, $\mathrm{Mcu}^{-/-}$mice were not protected from ischaemia-reperfusion-mediated heart injury when compared with wild-type mice. So, in the absence of MCU, it seems that calciumindependent cell death pathways might compensate.

Together, the results suggest that mitochondrial calcium uptake by MCU regulates stressed but not basal metabolic conditions. The precise role of MCU in tissue injury requires further study.

Kirsty Minton

ORIGINAL RESEARCH PAPER Pan, X. et al.

The physiological role of mitochondrial calcium revealed by mice lacking the mitochondrial calcium uniporter. Nature Cell Biol. http//dx.doi. org/10.1038/ncb2868 (2013) 